\title{
Re: Hysteropexy in the treatment of uterine prolapse stage 2 or higher: laparoscopic sacrohysteropexy versus sacrospinous hysteropexy - a multicentre randomized controlled trial (LAVA trial). (First comment on BJOG-19-1907.R1)
}

\author{
Min Huang ${ }^{1}$, Zi-Xi Loo ${ }^{2}$, Kun-Ling Lin ${ }^{2}$, and Cheng-Yu Long ${ }^{3}$ \\ ${ }^{1}$ Kuo General Hospital \\ ${ }^{2}$ Kaohsiung Medical University Hospital \\ ${ }^{3}$ Kaohsiung Municipal Siaogang Hospital
}

July 27, 2020

\section{Dear Editor,}

We really appreciate $\mathrm{MN}$ van Ijsselmuiden et al. for their efforts in conducting the first ever multicentre randomized controlled trial to compare laparoscopic sacrohysteropexy (LSH) versus sacrospinous hysteropexy (SSHP).$^{1}$ It has raised my queries and questions regarding the methodology and results of this trial. I would like to know the reasons to include the patients with history of previous pelvic floor or prolapse surgery in the exclusion criteria. If those above patients were randomly allocated into two surgical group equally, would it have impacts over the study result or design? Moreover, we are really interested and keen to know how to perform the anterior colporrhaphy and posterior colporrhaphy though laparoscopic method as mentioned in the article.

There are more patients presented with anterior vaginal wall prolapse: POP-Q stage- Aa or Ba $>0$ (LSH group:81\%; SSHP group:72.6\%) when compared to apical prolapse (LSH group:46.6\%; SSHP:45.6\%) in Table 1. It seems that the majority of study population had combined anterior and apical compartment prolapse but not with solely apical prolapse. Furthermore, Table 2 showed that overall anterior compartment failure rates are $50.9 \%$ and $56.9 \%$ in LSH group and SSHP group respectively in 1-year follow-up interval. The failure rate is extraordinarily high compared with previous study. ${ }^{2}$ Hysteropexy surgeries are useful to treat patients with sorely apical prolapse but not with patients with combine anterior and apical compartment prolapse with prominent cystocele. In controversy, a large majority were satisfied with the one year surgical results and would recommend the surgery to someone else (LSH: 87.7\%; SSHP: 89.7\%) even though there was a high recurrence rate of anterior wall prolapse in one year follow-up.

In the statistical analysis section, there were significantly more additional anterior vaginal wall repairs in the SSHP group compared to LSH group (SSHP: $\mathrm{n}=61,98.4 \%$; LSH: $\mathrm{n}=55,85.9 \%, \mathrm{P}=0.010$ ). I would like to know how this small number difference $(61-55=6)$ in these two groups can cause significant difference in $\mathrm{P}$ value and how to calculate this $\mathrm{P}$ value. This trial assumes a failure rate of $3 \%$ based on outcomes of SSHP in a previous prospective study. However, the data population is relatively small while the non-inferiority margin was set at $10 \%$.

The primary outcome is defined as a composite outcome of surgical failure of the apical compartment at 12 months' follow-up, defined as recurrence of uterine prolapse (POP-Q [?] stage 2). Surgical success was defined as no prolapse beyond the hymen. According to POP-Q stage system, POP-Q stage 2 is defined as most distal prolapse is between $1 \mathrm{~cm}$ above and $1 \mathrm{~cm}$ beyond hymen. ${ }^{3}$ The most prominent prolapse 
which descends beyond hymen would be also considered as stage 2 prolapse. It elicits clinical controversy and conflicts in the definition between surgical failure and success. We hope that this letter will deliver the message that precise preoperative patient selection and study design are crucial as they may have critical impacts over clinical outcomes and treatment success.

Min-syuan Huang, ${ }^{2,3}$ Zi-Xi Loo, ${ }^{1}$ Kun- Ling Lin,,${ }^{1,2}$ Cheng-Yu Long ${ }^{1,2}$

1. Department of Obstetrics and Gynecology, Kaohsiung Medical University Hospital, Kaohsiung Medical University, Kaohsiung, Taiwan

2. Graduate Institute of Medicine, College of Medicine, Kaohsiung Medical University, Kaohsiung, Taiwan

3. Department of Obstetrics and Gynecology, Kuo General Hospital, Tainan, Taiwan

\section{References}

1. van IMN, van Oudheusden A, Veen J, van de Pol G, Vollebregt A, Radder CM, et al. Hysteropexy in the treatment of uterine prolapse stage 2 or higher: laparoscopic sacrohysteropexy versus sacrospinous hysteropexy-a multicentre randomised controlled trial (LAVA trial). BJOG : an international journal of obstetrics and gynaecology. 2020.

2. Lucot JP, Cosson M, Bader G, Debodinance P, Akladios C, Salet-Lizée D, et al. Safety of Vaginal Mesh Surgery Versus Laparoscopic Mesh Sacropexy for Cystocele Repair: Results of the Prosthetic Pelvic Floor Repair Randomized Controlled Trial. European urology . 2018;74(2):167-76.

3. Bump RC, Mattiasson A, Bo K, Brubaker LP, DeLancey JO, Klarskov P, et al. The standardization of terminology of female pelvic organ prolapse and pelvic floor dysfunction. American journal of obstetrics and gynecology . 1996;175(1):10-7. 